\title{
The Kinematics of Hyper-Redundant Robot Locomotion
}

\author{
Gregory S. Chirikjian, Member, IEEE, and Joel W. Burdick
}

\begin{abstract}
This paper considers the kinematics of hyperredundant (or "serpentine") robot locomotion over uneven solid terrain, and presents algorithms to implement a variety of "gaits." The analysis and algorithms are based on a continuous backbone curve model which captures the robot's macroscopic geometry. Two classes of gaits, based on stationary waves and traveling waves of mechanism deformation, are introduced for hyper-redundant robots of both constant and variable length. We also illustrate how the locomotion algorithms can be used to plan the manipulation of objects which are grasped in a tentacle-like manner. Several of these gaits and the manipulation algorithm have been implemented on a 30 degree-of-freedom hyper-redundant robot. Experimental results are presented to demonstrate and validate these concepts and our modeling assumptions.
\end{abstract}

\section{INTRODUCTION}

"H YPER-REDUNDANT" robots have a large or infinite degree of kinematic redundancy. They are analogous in morphology to snakes, tentacles, and elephant trunks. Their high degree of articulation makes hyperredundant robots superior for operation in highly constrained environments, such as nuclear reactor cores, underground toxic waste tanks, or the human intestine. Many conceivable applications require the hyper-redundant robot to maneuver, via some form of locomotion, around its environment. This paper considers how to implement various forms of hyper-redundant robot locomotion.

Definition: Hyper-redundant robot locomotion is the process of generating net displacements of a hyper-redundant robotic mechanism via internal mechanism deformations. Actuatable wheels, tracks, or legs are not necessary.

Definition: A gait is a distinct repetitive cycle of mechanism deformation that leads to net robot displacement.

For a given mechanism, different gaits will have correspondingly different speed, robustness, and maneuverability characteristics. A gait which is well suited to one type of terrain or task may be ill-suited to another situation. For maximum adaptability, a hyper-redundant robot should be capable of switching between several different gaits types.

This paper considers the kinematics of two classes of gaits that can be used by hyper-redundant robots to locomote

Manuscript received September 9, 1992; revised June 17, 1993. This work was supported by the National Science Foundation under Grants MSS-901779 and MSS-9157843 and by the Office of Naval Research Young Investigator Award N00014-92-J1920.

G. S. Chirikjian is with the Department of Mechanical Engineering, Johns Hopkins University, Baltimore, MD 21218 USA.

J. W. Burdick is with the Department of Mechanical Engineering, California Institute of Technology, Pasadena, CA 91125 USA

IEEE Log Number 9414519. over uneven terrain. These gaits are based on stationary and traveling waves of mechanism deformation, and have analogues in inchworm and caterpillar locomotion and the creeping gaits of snakes. These gaits are largely "kinematic" in nature. That is, dynamic effects and a detailed model of the friction between the mechanism and the ground are not critical to the function or understanding of these gaits at reasonable speeds. In contrast, dynamic effects, the internal distribution of mechanism forces, and a frictional model are important for some gaits, such as those which are analogous to the undulatory and concertina gaits used by snakes. Hence, the analysis in this paper does not cover all possible hyperredundant robot gaits. The gaits considered in this paper were chosen for their simplicity, implementability, and wide range of applicability. Further, we show how these locomotion algorithms can be used to implement a novel scheme for planning the manipulation of objects which are grasped in a tentacle-like fashion.

The gait algorithms are based on a "backbone curve" modeling technique that was introduced in earlier works devoted to hyper-redundant manipulator kinematics, trajectory planning, and obstacle avoidance [5], [4], [8]. With the backbone curve abstraction, surprisingly simple ideas and mathematics can be used to understand and implement relatively complicated hyper-redundant robot locomotion phenomena. Several of the gaits and the object manipulation scheme have been implemented in a 30 degree-of-freedom hyper-redundant robot prototype, and experimental results are also presented to show that the algorithms are indeed able to be implemented.

\section{RELATION TO PRIOR WORK}

Inchworms, earthworms, snakes, and slugs have morphologies that can be considered hyper-redundant. Nature has evolved a diverse set of ways for these creatures to locomote. For example, slugs locomote via locomotory pulses, or pedal waves [17]. Snakes use three primary gait categories, termed the lateral undulatory, sidewinding, and concertina modes, whose qualitative properties have been widely studied [3], [16], [19]. Some varieties of snake also use "creeping gaits" during predation. This form of locomotion, which depends on a rhythmic expansion and contraction of the snake's muscles, has much in common kinematically with the movement of earthworms through soil [22], [25]. Many of these forms of undulatory biological locomotion can be idealized as traveling or stationary waves of body deformation. This observation guides our implementation of hyper-redundant robot locomotion gaits. The term "locomotion 


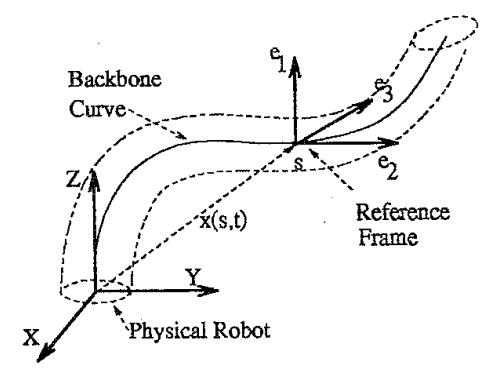

Fig. 1. Backbone curve.

wave" or "wave". will often be used to describe the mechanism deformations associated with these wave-like gaits. It should be stressed that this paper is not an analysis of biological locomotion, but is instead a study of how to implement hyper-redundant robot locomotion gaits that have biological counterparts.

In contrast to the largely empirical biological literature, Keller and Falkovitz [18] present a detailed mathematical analysis of worm locomotion. Their analysis only considers locomotion along a straight line which is induced by traveling waves of body contraction. Most of their paper is devoted to understanding the effects that friction and bounds on internal tension have on the maximum locomotion speed. In this paper, we consider a broader class of gaits than the one considered in [18], as well as locomotion on uneven terrain. Reference [18] also focuses on a particular model of mechanism actuation, and is thus limited in its applicability. We focus on the macroscopic kinematics of a broad class of gaits, and do not dwell on the mechanical structures and actuators required to implement the mechanism deformation.

Robotics engineers have investigated "snake-like" robots over the past quarter century. For a detailed history of hyperredundant robotic mechanisms, see [4]. Here we summarize prior activities which are most relevant to this study. The earliest hyper-redundant robot designs date to the late 1960's [2]. To our knowledge, the "active cord" mechanism of Hirose and Umetani [14] was the first hyper-redundant robotic system to successfully demonstrate locomotion. Since then, Hirose and co-workers have developed and demonstrated numerous mobile hyper-redundant mechanisms and mechanical morphologies, e.g., [13] (and references therein). While some of these hyper-redundant mobile robots are a hybrid between a snake-like vehicle and a wheeled vehicle, we consider locomotion schemes which do not rely on actuatable wheels, tracks, or legs. A conceptual scheme for locomotion of a Variable Geometry Truss (VGT) robot is discussed in [24], though no explicit analysis or algorithms for locomotion are given. The hyper-redundant robot prototype reviewed in Section IX is a planar VGT robot. Others have developed "inchworming" devices for crawling through pipes [12]. These devices use a locomotion scheme which is a subset of the longitudinal wave locomotion scheme presented in Section VI.

The prior literature on hyper-redundant robotic locomotion has focused more on system design than on analysis. This is in contrast to the extensive literature on static walking [23], dynamically stable walking and hopping [21], and wheeled vehicles [1]. We believe that the framework presented here is a step toward a more unified treatment of hyper-redundant robot locomotion that is applicable to a wide range of mechanical morphologies and situations.

\section{KInematics OF BACKBONE ReFERENCE SetS}

Our approach to kinematic modeling of hyper-redundant robots is based on a two-step modeling process. In the first step, we assume that regardless of mechanical implementation, the important macroscopic features of a hyper-redundant robot can be captured by a backbone curve. The backbone curve can be considered as the mechanism centerline or spine (Fig. 1). A backbone curve parametrization and an associated set of reference frames is collectively called the backbone reference set. The geometric aspects of hyper-redundant robot motion planning are reduced to the determination of the proper time varying behavior of the backbone reference set. We employ this engineering approximation and abstraction in order to focus on the salient geometric features of hyper-redundant locomotion.

In the second step of the backbone curve modeling approach, the continuous backbone curve geometry is used to specify the actual mechanism's joint displacements. The continuous backbone reference set specification can be used to directly determine the actuator displacements of a continuous morphology robot, such as one constructed from pneumatic actuator bundles. For discretely segmented morphologies, such as the VGT design described in Section IX, the continuous curve solution can be used, via a "fitting" process, to compute the actuator displacements which cause the manipulator to exactly assume or closely approximate backbone curve shape. An explanation of the fitting techniques which are used in subsequent examples can be found in [4] and [8].

The backbone curve modeling approach is a significant departure from traditional robot kinematic modeling techniques. A more extensive discussion of the advantages and suitability of using the backbone curve approach for modeling hyperredundant robots can be found in [4] and [8]. Here we review only the essential elements which are required for locomotion analysis.

In this paper we represent the Cartesian position of backbone curve points in the form

$$
\bar{x}(s, t)=\int_{0}^{s} l(\sigma, t) \bar{u}(\sigma, t) d \sigma
$$

where $s \in[0,1]$ is a dimensionless parameter that is a measure of distance along the backbone curve at time $t$. We define $s$ to be the normalized arclength of the backbone curve in a fixed reference state at time $t_{0}$. The normalized arclength at $t \neq t_{0}$ may differ from $s$ due to elongation or contraction of the backbone curve.

The backbone curve base is located at $s=0 . \bar{x}(s, t)$ is a vector from the backbone curve base to the backbone curve point at $s \cdot \bar{u}(s, t)$ is the unit tangent vector to the curve at $s$. $l(s, t)$ is the length of the curve tangent and assumes the form

$$
l(s, t)=1+\epsilon(s, t)>0 .
$$




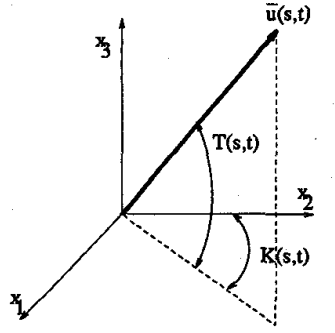

Fig. 2. Definition of $K(s, t)$ and $T(s, t)$.

$\epsilon(s, t)$ is the local extensibility of the backbone curve, which describes local expansion or contraction of the backbone relative to the fixed reference state at $t_{0}$. Depending upon the robot's mechanical implementation, the associated backbone curve may be inextensible $(\epsilon(s, t)=0 \forall s, t)$ or extensible. In the extensible case, the true arclength, $L$, at time $t$ is related to $l(s, t)$ via

$$
L(s, t)=\int_{0}^{s} l(\sigma, t) d \sigma
$$

otherwise, $L(s, t)=s$. Any parametrization of the unit sphere can be used to parametrize $\bar{u}(s, t)$ in (1). In this paper, we select

$$
\bar{u}(\cdot)=[\sin K(\cdot) \cos T(\cdot), \cos K(\cdot) \cos T(\cdot), \sin T(\cdot)]^{T}
$$

where $K(\cdot)$ and $T(\cdot)$ are Euler angles (Fig. 2). By convention, $K(0, t)=T(0, t)=0$ is assumed, so that $\bar{u}(0, t)=[0,1,0]^{T}$. The classical Frenet-Serret description of curves and this parametrization can be related as follows (where ( $)$ represents a derivative with respect to $s$ ):

$$
\begin{gathered}
\kappa^{2}=\frac{(\dot{T})^{2}+(\dot{K})^{2} \cos ^{2} T}{l^{2}} \\
\tau=\frac{1}{l}\left[\frac{\dot{K} \sin T-(\dot{T} \ddot{K}-\ddot{T} \dot{K}) \cos T-(\dot{T})^{2} \dot{K} \sin T}{\kappa^{2}}\right]
\end{gathered}
$$

where $\kappa$ and $\tau$ are the curvature and torsion of the backbone curve, respectively [10].

The kinematics of planar curves is a degenerate case of (4) with $T(s, t)=0 \forall s$. To distinguish the planar case, we use the symbol $\theta(s, t)$ instead of $K(s, t)$, where $\theta(s, t)$ is the clockwise measured angle which the tangent to the planar curve makes with the $x_{2}$-axis at time $t$. In this case, $\kappa(s, t)=(1 / l(s, t)) \partial \theta(s, t) / \partial s$, where $\kappa$ is positive for clockwise bending, and $\bar{u}(s, t)=[\sin \theta(s, t), \cos \theta(s, t)]^{T}$.

The backbone reference frame at $s$ has its origin coincident with $\bar{x}(s, t)$ (Fig. 1). The reference frame orientation can be expressed as: $\mathbf{Q}(s, t)=\left(\bar{e}_{1}(s, t) \bar{e}_{2}(s, t) \bar{e}_{3}(s, t)\right)$, where $\bar{e}_{j}(s, t)$ for $j=1,2,3$ are the reference frame basis vectors with respect to a frame at $s=0$, which is $\mathbf{Q}(0, t)=\mathbf{I}$ by convention. A given backbone curve parametrization will typically have a natural set of frames associated with it, in the same way that the Frenet-Serret frame is associated with curvature and torsion in the classical parametrization of curves. We call these induced reference frames. When the backbone curve is parametrized by $K(s, t)$ and $T(s, t)$, the matrix represents a unique induced reference frame orientation at each $(s, t)$ (see (6) at the bottom of the page). $\mathbf{Q}(s, t)$ may differ from $\mathbf{Q}_{I R}(s, t)$ by an $s$-dependent twist about the backbone curve tangent, which we term the roll distribution, $R(s, t)$. It is defined as: $\mathbf{Q}(s, t)=\operatorname{Rot}(\bar{u}, R(s, t)) \mathbf{Q}_{I R}$, where $\operatorname{Rot}(\bar{v}, \phi)$ is a rotation about vector $\bar{v}$ by angle $\phi$. For consistency with previous work, the second column of $\mathbf{Q}$ is chosen to be the backbone curve tangent vector, $\bar{u}(s, t)=\bar{e}_{2}(s, t)$.

In summary, the backbone reference set, which describes the important macroscopic geometry of a hyper-redundant robot, depends on a set of "shape functions," which we denote as $\left\{\mathcal{S}_{i}\right\}$. In this work, we choose the functions $l(s, t)$, $K(s, t), T(s, t)$, and $R(s, t)$, though other choices are possible. $R(s, t)$ is neglected in this paper because the kinematics of the gaits described below can be described without regard to the function $R(s, t)$ for robots with axially symmetric cross sections.

\section{TERRAIN MODEL}

The path $\bar{P}(\mu)=\left[P_{1}(\mu), P_{2}(\mu), P_{3}(\mu)\right]^{T}$ traversed by the mobile robot is assumed to be a once-differentiable arclength parameterized curve lying in the terrain surface where $\mu$ is the terrain arc-length parameter. ${ }^{1}$ With these assumptions, the path can be parametrized (in the form of (1) and (4)) by $l_{P}(s, t)=1$ and functions $K_{P}(\mu)$ and $T_{P}(\mu)$, where the subscript $P$ indicates that these functions refer to the path curve, and not the backbone curve. A fixed reference frame can be defined as follows. Assume the robot is lying at rest on the terrain at $t=0$. Let the backbone curve base $(s=0)$ be over terrain point $\bar{P}\left(\mu_{0}\right)$ (Fig. 3). We choose $\bar{P}\left(\mu_{0}\right)$ to be the origin of a fixed reference frame, $F_{0}$. The basis vectors of $F_{0}$ are assigned using the induced frame in $(6)$, with $K(s, t)$ and $T(s, t)$ replaced by $K_{P}(\mu)$ and $T_{P}(\mu) . \mu_{r}(t)$ denotes the location of the robot's rear at time $t$, where $\mu_{r}\left(t_{0}\right)=\mu_{0}$.

Hereafter, the physical girth of the robot is ignored. In practice, the robot's thickness displaces the backbone curve from the path curve. This effect can be accounted for using the theory of "offset curves" [11]. Because we consider only kinematic, and not dynamic, issues in this paper, we further assume that when necessary there is sufficient friction between the robot and the ground to preclude unwanted lateral sliding.

Let $s_{b}(t)$ and $s_{f}(t)$ respectively denote the value of the backbone curve parameter at the "back" and "front" of a

${ }^{1}$ For some gaits, only piecewise continuity with a finite number of nondifferentiable points is required.

$$
\mathbf{Q}_{I R}(s, t)=\left(\begin{array}{ccc}
\cos K(s, t) & \sin K(s, t) \cos T(s, t) & -\sin K(s, t) \sin T(s, t) \\
-\sin K(s, t) & \cos K(s, t) \cos T(s, t) & -\cos K(s, t) \sin T(s, t) \\
0 & \sin T(s, t) & \cos T(s, t)
\end{array}\right)
$$




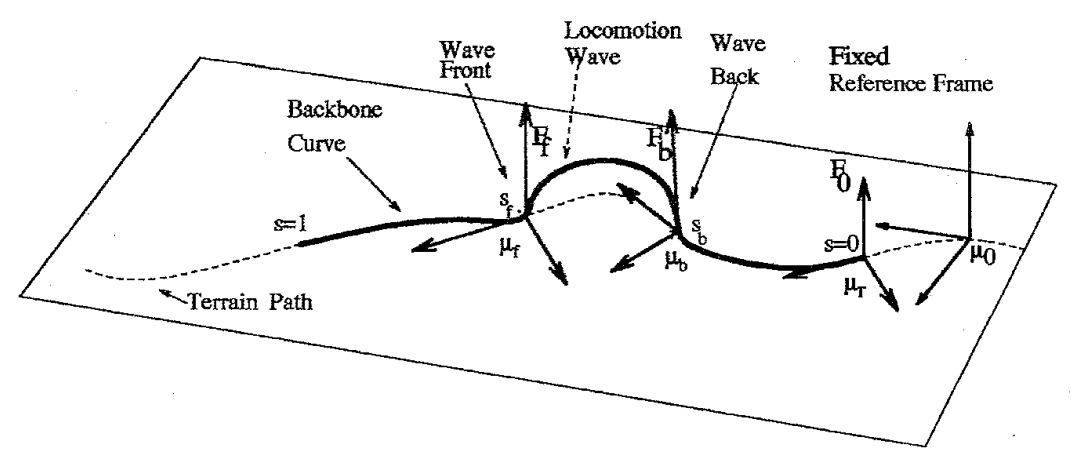

Fig. 3. Terrain model.

locomotion wave (Fig. 3). Let $\bar{P}\left(\mu_{b}(t)\right)$ and $\bar{P}\left(\mu_{f}(t)\right)$ denote the path points coincident with the points $s_{b}(t)$ and $s_{f}(t)$ in the backbone. For convenience, let $F_{r}, F_{b}$, and $F_{f}$ respectively denote the path frames at $\mu_{r}, \mu_{b}$, and $\mu_{f}$. Similarly, let $\widetilde{F}_{r}$, $\widetilde{F}_{b}$, and $\widetilde{F}_{f}$ denote the backbone reference frames at $s=0$, $s_{b}(t)$, and $s_{f}(t)$.

We can now define a principal problem to be solved in the ensuing sections. The segment(s) involved in the locomotion wave(s) should assume a shape which does not intersect the terrain and which smoothly blends with the terrain at the ends of the locomotion wave segment-i.e., the displacement of $\widetilde{F}_{f}$ with respect to $\widetilde{F}_{b}$ should match the displacement of $F_{f}$ with respect to $F_{b}$. However, the relative displacement of $F_{f}$ with respect to $F_{b}$ varies as the locomotion wave moves over the uneven terrain. Our goal is to determine how the backbone curve shape should vary with time in order to effect this terrain tracking for a variety of gaits. We call this the terrain matching problem. The solution to the terrain matching problem is sufficient to solve the geometric aspects of hyper-redundant locomotion planning.

With this terrain model in mind, we introduce a definition of stride length.

Definition: The stride length, $L_{S L}$, which is normally the distance traveled by a legged robot over one cycle of foot placements, is defined as the path arclength traversed by the robot during one cycle of mechanism deformation.

\section{LOCOMOTION GATTS FOR INEXTENSIBLE MECHANISMS}

We divide our study of gaits into those suitable for inextensible and extensible mechanisms. For the inextensible case considered in this section, body deformation is restricted to bending. We first consider inextensible traveling wave gaits, which have counterparts in slug pedal waves, caterpillar locomotion, and snake creeping gaits. We then consider inextensible stationary wave gaits, which are reminiscent of the gait used by inchworms.

\section{A. Inextensible Traveling Wave Locomotion Gaits}

An inextensible traveling wave gait can be implemented by transmitting a wave of bending deformation along the backbone curve. We restrict the locomotion wave to a backbone curve segment with constant arc-length, denoted by $L_{w} \ll 1$. Thus, $L_{w}=s_{f}(t)-s_{b}(t)$. Similarly, let $L_{P}(t)=\mu_{f}(t)-\mu_{b}(t)$ be the path arc-length between the points at which the front and back of the locomotion wave contact the terrain. The stride length is thus: $L_{S L}=L_{w}-L_{P}$. In the following algorithm, $L_{S L}$ is constant for every gait cycle.

Let the locomotion wave travel along the backbone curve with constant speed $\omega$, i.e., $\dot{s}_{f}=\dot{s}_{b}=\omega$ (where ( $)$ now represents a derivative with respect to time). If the backbone curve properly tracks the terrain, $\mu_{f}(t)$ and $\mu_{b}(t)$ also move along the terrain with speed $\omega$. Terrain tracking for traveling wave locomotion can be implemented if the backbone curve shape functions take the general form

$$
\begin{aligned}
K(s, t)= & K_{P}\left(\mu_{r}(t)+s\right) W\left(s, 0, s_{b}(t)\right) \\
& +K_{\text {wave }}\left(s-\alpha_{t}(t), t\right) W\left(s, s_{b}(t), s_{f}(t)\right) \\
& +K_{P}\left(\mu_{r}(t)+s-L_{S L}\right) W\left(s, s_{f}(t), 1\right) \\
T(s, t)= & T_{P}\left(\mu_{r}(t)+s\right) W\left(s, 0, s_{b}(t)\right) \\
& +T_{\text {wave }}\left(s-\alpha_{t}(t), t\right) W\left(s, s_{b}(t), s_{f}(t)\right) \\
& +T_{P}\left(\mu_{r}(t)+s-L_{S L}\right) W\left(s, s_{f}(t), 1\right)
\end{aligned}
$$

where

$$
\begin{aligned}
& \alpha_{t}(t)=\omega\left[t-I\left(\frac{t, 1+L_{w}}{\omega}\right)\right]-L_{w} \\
& W\left(s, s_{1}, s_{2}\right)= \begin{cases}1, & s \in\left[s_{1}, s_{2}\right) \\
0, & \text { otherwise. }\end{cases}
\end{aligned}
$$

$s_{b}(t)=\alpha_{t}(t)$, and $s_{f}(t)=s_{b}(t)+L_{w} . I(t, b)=b\lfloor t / b\rfloor$, where $\lfloor\cdot\rfloor$ is the greatest integer function. $W\left(s, \dot{s}_{1}, s_{2}\right)$ is a termed a "window function." The first and third terms of the shape functions in (7) ensure that the backbone curve segments $s \in\left[0, s_{b}(t)\right]$ and $s \in\left[s_{f}(t), 1\right]$ rest on the terrain. The second term implements the locomotion wave in the segment $s \in\left[s_{b}, s_{f}\right]$. The function $\alpha_{t}(t)$ in (8) linearly increases with time and is "reset" after a period $\left(1+L_{w}\right) / \omega$. This causes the locomotion wave to travel at constant speed $\omega$ from the rear of the robot to the front. After the wave "exits" the front of the backbone curve, another wave is subsequently generated at the rear of the robot, and the cycle repeats. Thus, the average wave speed is $(\omega) /\left(1+L_{w}\right)$, and every cycle causes the robot to be displaced by a distance $L_{S L}$ along the path. Alternatively, multiple waves can be used to increase the net robot speed for a given $\omega$. 


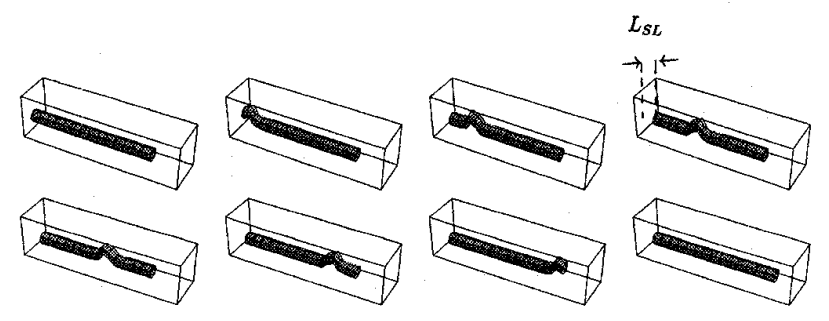

Fig. 4. Snapshots of a simulation of inextensible traveling wave locomotion gait. One complete cycle is shown.

For the locomotion wave to smoothly track the terrain, $K_{\text {wave }}(s, t)$ and $T_{\text {wave }}(s, t)$ must be chosen so that the displacement of $\tilde{F}_{f}(t)$ relative to $\tilde{F}_{b}(t)$ matches the displacement of $F_{f}(t)$ relative to $F_{b}(t)$. Let $\Delta \bar{X}^{\text {des }}(t)$ denote the displacement of $F_{f}$ relative to $F_{b}$. Assuming that the robot's cross section is radially symmetric about the backbone curve tangent, $F_{f}$ will be displaced relative to $F_{b}$ with 5 independent degrees of freedom (DOF). Using the backbone curve parametrization resulting from (4), $\Delta \bar{X}^{\mathrm{des}}(t)$ takes the form

$$
\Delta \bar{X}^{\mathrm{des}}(t)=\left[\begin{array}{c}
P_{1}\left(\mu_{f}(t)\right)-P_{1}\left(\mu_{b}(t)\right) \\
P_{2}\left(\mu_{f}(t)\right)-P_{2}\left(\mu_{b}(t)\right) \\
P_{3}\left(\mu_{f}(t)\right)-P_{3}\left(\mu_{b}(t)\right) \\
K_{P}\left(\mu_{f}(t)\right)-K_{P}\left(\mu_{b}(t)\right) \\
T_{P}\left(\mu_{f}(t)\right)-T_{P}\left(\mu_{b}(t)\right)
\end{array}\right] .
$$

Conceptually, the terrain matching problem is equivalent to a hyper-redundant manipulator inverse kinematic, or "hyperredundancy resolution" problem. That is, the locomotion wave backbone curve segment can be thought of as the backbone curve of a hyper-redundant robot manipulator (with length $L_{w}$ ) whose base reference frame is aligned with $F_{b}(t)$ and whose tip frame must align with $F_{f}(t)$. The authors have previously developed schemes for solving this problem, using the kinematic modeling scheme of Section III.

In one approach [9], the calculus of variations is used to find the shape functions which cause the manipulator (here, the locomotion wave segment) to satisfy necessary boundary conditions (the terrain matching problem) while also minimizing a user defined criteria, such as the total bending of the backbone curve. While this approach is good for selecting locomotion wave shapes which minimize some criteria, such as mechanism bending or actuator forces, it is less attractive for locomotion applications. A conceptually and computationally simple approach, based on a "modal" selection of the shape functions, is discussed in [8]. We shall use this method here for the purposes of illustration.

In the modal approach, the backbone curve shape functions that control the locomotion wave segment are arbitrarily restricted to a "modal" form

$$
\mathcal{S}_{\text {wave }_{i}}(\sigma, t)=\sum_{j=1}^{N} a_{i}(t) \phi_{j}\left(\frac{\sigma}{L_{w}}\right) \text { where } \sigma=s-\alpha_{t}(t) \text {. }
$$

The $\left\{\phi_{j}(\cdot)\right\}$ are termed mode functions, while the $\left\{a_{j}(t)\right\}$ are termed modal participation factors. In other words, we arbitrarily restrict the shape functions to be the sum of a specified set of primitive functions. The $\left\{\phi_{j}\right\}$ are chosen by the hyper-redundant robot programmer, and often reflect the physical characteristics of the problem. The $\left\{\phi_{j}\right\}$ must satisfy independence and nondegeneracy conditions [8]. $N_{\mathcal{S}_{i}}$ is the number of modes used to define the $i$ th shape function, and the total number of modes must equal or exceed the number of terrain matching constraints. The set of modal participation factors is hereafter denoted by $\bar{a}(t) \in \mathbb{R}^{\sum_{i} N_{\mathcal{S}}}$.

Let $\Delta X(\bar{a}(t), t)$ denote the displacement of $\tilde{F}_{f}(t)$ with respect to $\tilde{F}_{b}(t)$ when the locomotion wave shape functions are restricted to a modal form. For terrain matching, the goal is to determine $\bar{a}(t)$ so that

$$
\Delta \bar{X}(\bar{a}(t), t)=\Delta \bar{X}^{\text {des }}(t) .
$$

Note that the terrain matching problem can also be put in this same form when employing the other hyper-redundancy resolution schemes considered in [9] if $\bar{a}$ is interpreted as a set of "reduced" curve coordinates. In the optimal shape function method, $\bar{a}$ would be the Lagrange multipliers associated with isoperimetric end-effector positioning constraints. Thus, (12) is a general statement of the terrain matching computational problem. While (12) arose from the consideration of the traveling wave gait, the terrain matching problem also takes this same form for the other gaits described below, where the forms of $\Delta \bar{X}^{\text {des }}$ and $\Delta \bar{X}$ depend on the chosen gait.

Let us first consider an example of a straight line path lying in the plane. We often consider the straight line locomotion case in this paper because of its relevance to the experiments in Section IX. The inextensible backbone curve is restricted to a plane, and thus its geometry can be captured using the single shape function $\theta(s, t)$, where $\theta(s, t)$ is defined so that the $x_{2^{-}}$ axis is terrain, while the $x_{1}$-axis is the outward normal to the terrain. Assume that $\theta(s, t)$ takes a modal form with single mode $\phi_{t}(\cdot)$ and a single modal participation factor $a_{t}(t)$. It can be shown that any $\phi_{t}(s), a_{t}(t)$ satisfying the constraints

$$
\begin{gathered}
\phi_{t}\left(s_{b}\right)=\phi_{t}\left(s_{f}\right)=0 \\
\int_{s_{b}}^{s_{f}} \cos \left(a_{t} \phi_{t}(\sigma)\right) d \sigma=0 ; \quad \int_{s_{b}}^{s_{f}} \sin \left(a_{t} \phi_{t}(\sigma)\right) d \sigma \geq 0
\end{gathered}
$$

will insure proper terrain matching and prevent the locomotion wave from penetrating the terrain if the associated modal participation factor is within a reasonable range of values. One of the infinite number of functions that satisfy (12) and (13) is

$$
\theta(s, t)=\frac{L_{w} C_{b}}{2 \pi} W\left(s-\alpha_{t}(t), 0, L_{w}\right) \sin \frac{2 \pi}{L_{w}}\left(s-\alpha_{t}(t)\right)
$$

where $C_{b}$ is the maximum backbone curvature in the locomotion wave segment and $\alpha_{t}(t)$ takes the form of (8). A simulation of this locomotion, employing (14) and (8), is shown in Fig. 4, for the case $L_{w}=1 / 4$. For this example, $L_{S L}$ is the difference between the locomotion wave segment arc-length and the "footprint" of the wave on the terrain. It can be shown that

$$
L_{S L}=L_{w}\left[1-J_{0}\left(\frac{C_{b} L_{w}}{2 \pi}\right)\right]
$$

where $J_{0}$ is the zeroth order Bessel function. 
It is easy to find closed form solutions to (12) for straight line locomotion. It is generally not possible to solve (12) for $\bar{a}(t)$ in closed form when operating on uneven terrain. A number of numerical techniques can be employed to solve these nonlinear equations. Here we review a simple method that is analogous to the popular resolved rate trajectory planning scheme (which is essentially Newton iteration). It is quite useful in practice. Similar schemes exist for other the hyper-redundancy resolution approaches.

This scheme is based on the time rate-of-change of (12),

$$
\Delta \dot{\bar{X}}^{\text {des }}=\mathcal{J}(\bar{a}) \dot{\bar{a}}+\frac{\partial \Delta \bar{X}(\bar{a}, t)}{\partial t},
$$

where $\mathcal{J}(\bar{a})=\partial \Delta \bar{X}(\bar{a}, t) / \partial \bar{a}$ is the modal Jacobian matrix. The term $\partial \Delta \bar{X}(\bar{a}, t) / \partial t$ accounts for the explicit dependence of $\Delta \bar{X}(\bar{a}, t)$ on time due to the relative motion of the locomotion wave along the curve. $\mathcal{J}(\bar{a})$ can be obtained by differentiating (1) when the shape functions are restricted to the modal form, (11). Depending upon the choice of modes, the elements of $\mathcal{J}(\bar{a})$ may be computed symbolically, or using Liebniz's rule and numerical integration.

In the context of the terrain matching problem, (16) can be used in two ways. First, (16) can be used to numerically solve for $\bar{a}\left(t_{0}\right)$ at a given time, $t_{0}$. Let $\tilde{\bar{a}}_{0}\left(t_{0}\right)$ be an estimate of $\bar{a}\left(t_{0}\right)$, which is assumed to be "near" $\bar{a}\left(t_{0}\right)$. (16) can be iterated in a differential form,

$$
\begin{aligned}
\tilde{\bar{a}}_{k+1}\left(t_{0}\right)=\tilde{\bar{a}}_{k}\left(t_{0}\right)+\Delta t \mathcal{J}^{-1}\left(\tilde{\bar{a}}_{k}\left(t_{0}\right)\right) \\
\cdot\left[\Delta \bar{X}^{\text {des }}\left(t_{0}\right)-\Delta \bar{X}\left(\tilde{\bar{a}}_{k}\left(t_{0}\right)\right)-\frac{\partial \Delta \bar{X}\left(\tilde{\bar{a}}_{k}\left(t_{0}\right)\right)}{\partial t}\right]
\end{aligned}
$$

to find $\bar{a}\left(t_{0}\right) \cdot \mathcal{J}^{-1}(\cdot)$ is a generalized modal Jacobian inverse and $\tilde{\bar{a}}_{k}\left(t_{0}\right)$ is the estimated value of $\bar{a}$ at the $k$ th iteration of (17). For "small" $\Delta t,(17)$ will converge, assuming that $\mathcal{J}$ is not singular. Conditions under which "modal singularities" occur are discussed in [8]. The second way (16) can be used is to effect terrain tracking. $\Delta \dot{\bar{X}}^{\text {des }}(t)$ is computed from the terrain path and a traveling wave speed $\left(\Delta \bar{X}^{\text {des }}(t)\right.$ will take other forms for other gaits). Assume $\bar{a}\left(t_{0}\right)$ is known, and perhaps has been found using the technique described above. Equation (16) is then solved for $\dot{\bar{a}}(t)$, which is then numerically integrated to determine the values of $\bar{a}(t)$ which cause terrain tracking.

This numerical method generally requires the computation of a $5 \times N_{T}$ modal Jacobian matrix and its inverse. If the chosen modes do not have a closed form forward kinematic solution, then all $5 N_{T}$ elements of the modal Jacobian must be computed using numerical integration. This may make real time computation marginally feasible with limited computational power. However, we can use the following "trick" to simplify the computational aspects of this problem. Let $K_{\text {wave }}$ and $T_{\text {wave }}$ be restricted to the forms

$$
\begin{aligned}
\mathcal{S}_{\text {wave }_{i}}(\xi, t)= & g(\xi) \mathcal{S}_{P_{i}}\left(\mu_{f}(t)\right)+(1-g(\xi)) \mathcal{S}_{P_{i}}\left(\mu_{b}(t)\right) \\
& +\sum_{j=1}^{N} a_{j} a_{j}(t) \hat{\phi}_{j}(\xi)
\end{aligned}
$$
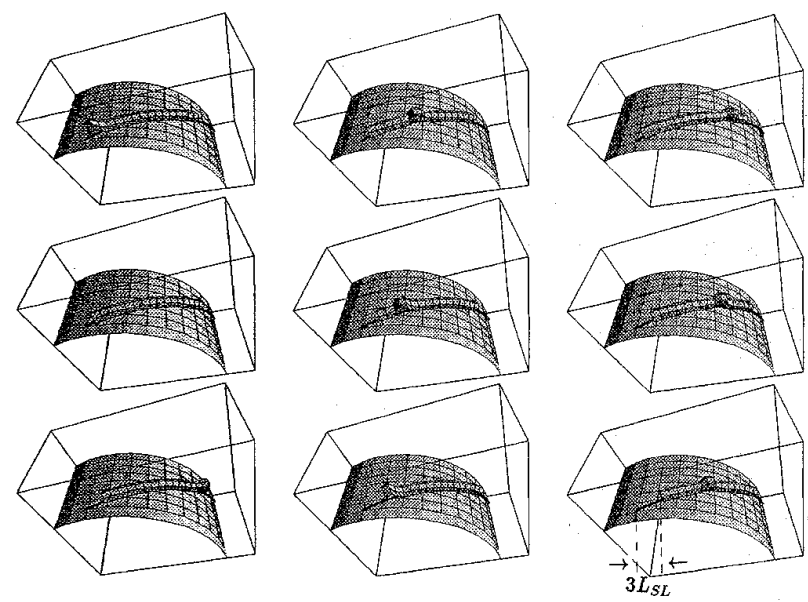

Fig. 5. Snapshots of traveling wave locomotion simulation. The robot follows a helical path over a cylindrical terrain.

where $\mathcal{S}_{P_{1}}=K_{P}, \mathcal{S}_{P_{2}}=T_{P}$, and $\xi=\left(s-s_{b}\right) / L_{w}$. $g(\xi)$ is a monotonically increasing function with $g(0)=0$ and $g(1)=1$. The mode functions are restricted to assume zero value at $\xi=0,1$. In this way, the backbone curve tangency conditions are automatically met at $s_{b}$ and $s_{f}$. Thus, in general only three modal participation factors, which control the displacement of the origin of $F_{f}$ with respect to $F_{b}$, are required. Hence, the modal Jacobian need at most be a $3 \times 3$ matrix, though extra modes can be added. This simplifies practical computation.

Fig. 5 shows an example of locomotion along a helical path over a cylindrical terrain. In this case, $\bar{P}(\mu)=$ $[r \cos (\mu / \eta), r \sin (\mu / \eta), B \mu / \eta]^{T}$, where $r$ is the radius of the cylindrical terrain (1.0 in this example), $B$ is the helix pitch (0.4 in this example), and $\eta=\sqrt{r^{2}+B^{2}}$. For this terrain, $K_{P}=-\mu / r$ and $T_{P}=\operatorname{Atan} 2(r / \eta, B / \eta)$, where $\operatorname{Atan} 2$ is the four quadrant, or polar, inverse tangent function. In this case, $K_{\text {wave }}(s, t)$ and $T_{\text {wave }}(s, t)$ take the form of (18). Two modes, $\hat{\phi}_{1}(\xi)=\sin (2 \pi \xi)$ and $\hat{\phi}_{2}(\xi)=\sin (3 \pi \xi)$, are assigned to $K_{\text {wave }}$, while one mode, $\hat{\phi}_{3}(\xi)=\sin (\pi \xi)$, is assigned to $T_{\text {wave }}$. Another related example is shown in the next section.

Many other mode choices are possible in the above examples, and it should be stressed that the method is not highly sensitive to the particular choice of mode functions. Alternatively, one can use other hyper-redundancy resolution methods which are not dependent upon the intuitive choice of modes.

\section{B. Inextensible Stationary Wave Locomotion Gaits}

Inextensible stationary wave gaits rely upon a body-fixed backbone curve shape whose amplitude of bending oscillates. Roughly speaking, this is analogous to the way an inchworm extends and contracts its body so that the "hump" or "loop" remains in approximately the same body location. To illustrate this gait, let us again consider straight line locomotion over flat terrain. Inextensible stationary wave locomotion will result if $\theta(s, t)$ takes the form

$$
\theta_{s}(s, t)=a_{s}(t) \phi_{s}\left(s-s_{b}\right) W\left(s, s_{b}, s_{f}\right)
$$



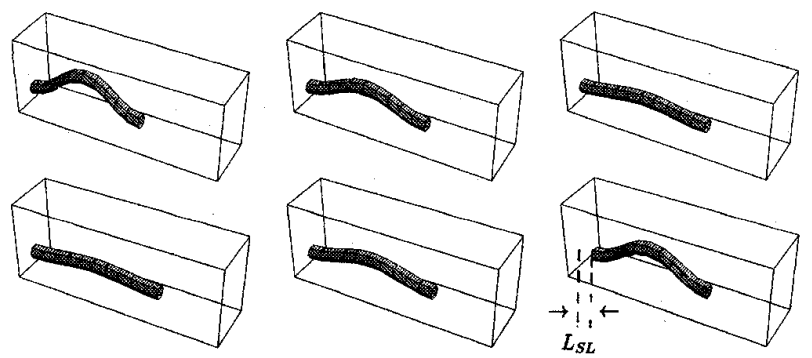

Fig. 6. Inextensible stationary wave locomotion gait: $m=1$.

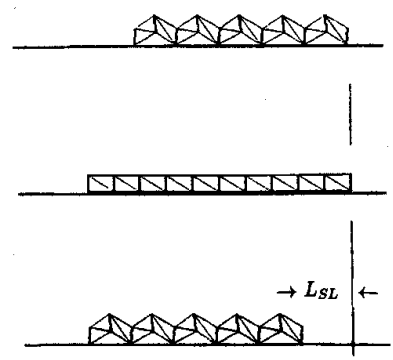

Fig. 7. Inextensible stationary wave locomotion gait: $m=5$.

where $a_{s}(t)$ is a periodic function, and $\left\{s_{b}, s_{f}\right\}$ are constant. In this way, the locomotion wave, which is restricted to a fixed backbone curve segment of length $L_{w}$ in the interval $s \in\left[s_{b}, s_{f}\right]$, oscillates in amplitude while retaining its relative position in the robot.

Remark: Because we are using only a kinematic (not a dynamic) model, this stationary wave locomotion model implicitly assumes that the mechanism has less resistance to motion in the forward direction than in the reverse direction. In nature, this effect is achieved by scales which slide during forward motion and exert traction on the ground preventing retrograde motion. In Section IX we demonstrate a mechanical device which implements this characteristic.

In the flat terrain case, any function of the form (19) where $\phi_{s}(\cdot)$ and $a_{s}(t)$ satisfy constraints of the form (13) is a suitable solution to the terrain matching problem. One such choice is

$$
\phi_{s}(s)=\frac{L_{w}}{2 \pi} \sin \left(\frac{2 \pi\left(s-s_{b}\right)}{L_{w}}\right) \quad a_{s}(t)=a_{0}+a_{1} \cos \omega t
$$

for some wavelength $L_{w} \cdot a_{0}$ and $a_{1}$ in (20) must obey $a_{\max }>a_{0}>\left|a_{1}\right|>0$, where $a_{\max }$ is the maximum allowable backbone curvature and $a_{0}>\left|a_{1}\right|>0$ guarantees that $\phi_{s}(s)$ satisfies (13). Fig. 6 illustrates this solution for the case $L_{w}=1$, i.e., $s_{b}=0$ and $s_{f}=1$. With the contact friction assumption described above, the stride length in this case is the difference between the maximum and minimum nominal body length over one cycle:

$$
L_{S L}=J_{0}\left(\frac{L_{w}\left(a_{0}-a_{1}\right)}{2 \pi}\right)-J_{0}\left(\frac{L_{w}\left(a_{0}+a_{1}\right)}{2 \pi}\right)
$$

Fig. 7 shows this same gait for the case $L_{w}=1 / 5$. A variable geometry truss (VGT) has been fit to the backbone curve for clarity. In practice, choosing $L_{w} \ll 1$ lowers the robot center of gravity and increases the stride length.
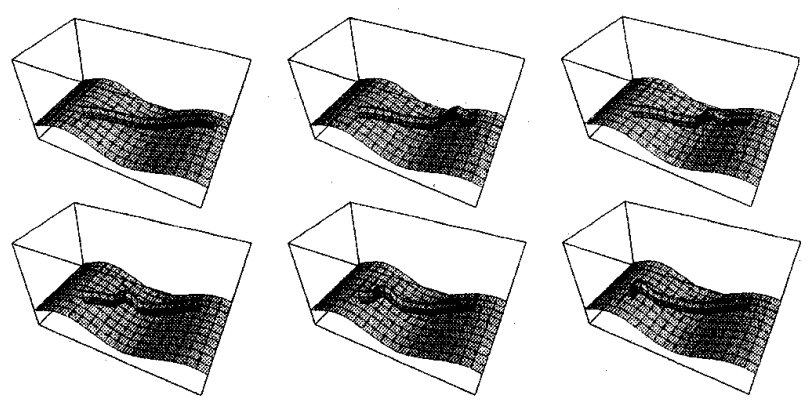

Fig. 8. Gait employing a stationary wave cycle and a traveling wave cycle.

The generalization of this gait to curvilinear paths over uneven terrain is based on a more general form for $\Delta \bar{X}^{\text {des }}$. In brief, we need to find expressions for $\mu_{b}(t)$ and $\mu_{f}(t)$, which cause those points to alternately slide forward along the terrain in a fashion which is analogous to the way that the contact points between the robot and the terrain slide in the simple straight line standing wave gait above. This can be done if $\mu_{b}(t)$ and $\mu_{f}(t)$ take the forms

$$
\begin{gathered}
\mu_{b}(t)=L_{S L}\left[I\left(t, t_{p}\right)+\beta_{1}\left(t-I\left(t, t_{p}\right)\right)\right. \\
\left.\times W\left[t-I\left(t, t_{p}\right), 0, \frac{t_{p}}{2}\right]\right]+\mu_{0} \\
\mu_{f}(t)=L_{S L}\left[I\left(t, t_{p}\right)+\beta_{2}\left(t-I\left(t, t_{p}\right)\right) \times\right. \\
\left.W\left[t-I\left(t, t_{p}\right), \frac{t_{p}}{2}, t_{p}\right]\right]+\mu_{0}+L_{w}-L_{S L}
\end{gathered}
$$

where $t_{p}$ is the period of the stationary wave oscillation. $\beta_{1}(\cdot)$ is any function with the property that $\beta_{1}(0)=0$ and $\beta_{1}\left(t_{p} / 2\right)=1$. For example, $\beta_{1}(t)=\cos \left(\frac{\pi\left(t_{p} / 2-t\right)}{t_{p}}\right)$ is a suitable choice. Similarly, $\beta_{2}(\cdot)$ is any function such that $\beta_{2}\left(t_{p} / 2\right)=0$ and $\beta_{2}\left(t_{p}\right)=1 . \beta_{2}(t)=\cos \left(\frac{\pi\left(t_{p}-t\right)}{t_{p}}\right)$ is one possible choice.

In this way, the point $\mu_{b}(t)$ is moving forward along the ground during the first half of the oscillation phase, while $\mu_{f}(t)$ is stationary. Similarly, during the second half of the oscillation period, $\mu_{b}(t)$ is stationary, while $\mu_{f}(t)$ moves forward. Substituting (22) into (10) results in a suitable $\Delta \bar{X}^{\text {des }}(t)$ for use in (12). Since we are assuming inextensibility, the shape of the stationary locomotion wave will oscillate in a way analogous to (19). The particular choice of the functions $\beta_{1}(\cdot)$ and $\beta_{2}(\cdot)$ determines the actual sliding velocity of $\mu_{f}$ and $\mu_{b}$ during their respectively active periods.

The generalized stationary wave gait may be less useful in practice than the traveling wave gait as it assumes that the portions of the mechanism which are not part of the locomotion wave slide along the path. This may be difficult for paths with high curvature. However, the generalized stationary wave gait can be useful in the following way. Fig. 8 depicts snapshots of a computer simulation of a locomotion scheme which uses one half of a stationary wave locomotion cycle to generate a wave shape at the rear of the robot. This wave then traverses the length of the robot using a bending traveling wave, and subsequently exits the front of the manipulator using the second half of the stationary wave cycle. 

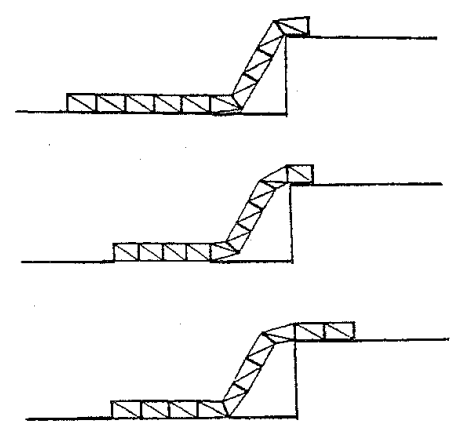

Fig. 9. Extensible locomotion over a step.

\section{LOCOMOTION GAITS FOR EXTENSIBLE MECHANISMS}

In addition to the gaits considered in the last section, extensible mechanisms, such as the VGT structure considered in Section IX, can employ stationary and traveling locomotion waves of mechanism extension, or combinations of extension and bending. This section considers the extensible counterparts of the gaits described in the previous section.

Purely extensible locomotion can be implemented by constraining the backbone curve to the form

$$
\bar{x}(s, t)=\bar{P}\left(L(s, t)+\mu_{r}(t), t\right) .
$$

That is, net motion is generated by sliding portions of the mechanism along the ground in a repetitive wave-like manner. The choice of $L(s, t)$ (or equivalently, $l(s, t)$ ) determines the particular locomotion gait. $\mu_{r}(t)$ will similarly be determined by the chosen gait.

A longitudinal stationary wave gait results when $l(s, t)$ assumes the form

$$
l(s, t)=a_{s}(t) \phi_{s}(s)
$$

where $a_{s}(t)$ is periodic. $\phi_{s}$ controls how the elongation is distributed throughout the mechanism. Fig. 9 shows how this gait can be used to effect locomotion over a step. The path in this case consists of three segments: a horizontal ground line, a fictitious "ramp" connecting the ground and the top of the step, and line representing the top of the step. In this example, $l(s, t)$ is defined as in (24) with

$$
a_{s}(t)=1-\frac{L_{S L}}{2} \cos \left(\frac{2 \pi t}{t_{p}}\right) \quad \phi_{s}(s)=1 .
$$

It can be shown that

$$
\mu_{r}(t)=L_{S L}\left[\mathrm{I}\left(t, t_{p}\right)+\cos ^{2}\left(\pi t / t_{p}\right) W\left(t-I\left(t, t_{p}\right), \frac{t_{p}}{2}, t_{p}\right)\right]
$$

where $t_{p}$ is the gait period. For this example, $L_{S L}=0.1$ and $t_{p}=1$. A VGT has again been fit to the backbone curve to more clearly illustrate the sequence of mechanism deformation during the gait.

Similarly, a longitudinal traveling wave gait results if

$$
l(s, t)=1+\alpha_{l}(t) \phi_{l}\left(s-\alpha_{t}(t)\right) W\left(s-\alpha_{t}(t), 0, L_{w}\right)
$$

where $\alpha_{t}(t)$ assumes a form similar to (8). In this gait, a wave (confined to a backbone curve segment of referential length
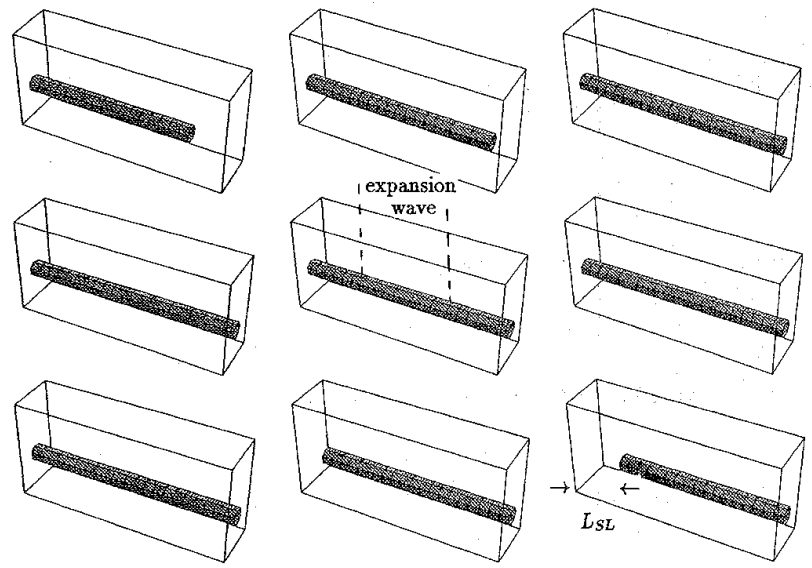

Fig. 10. Extensible traveling wave gait. An expansion wave travels from the front to the rear of the mechanism as the mechanism moves to the right.

$L_{w}$ ) of mechanism expansion or contraction travels the length of the backbone. This pattern of mechanism deformation is analogous to the wave-like muscle contractions of peristalsis. The distribution of the elongation within the wave is determined by $\phi_{l}(\cdot)$. Fig. 10 shows a simulation of this gait over a straight line path for the case $\theta(s, t)=0, L_{w}=1 / 4$, and $l(s, t)=1+E_{l} \phi_{l}\left(s-\alpha_{t}(t)\right)$, where $\phi_{l}(\cdot)=W(\cdot, 0,1 / 5)$ and $E_{l}=1$. Note that this is an expansion wave, which must travel in the direction opposite to the direction of robot travel. If $E_{l}<0$, the locomotion wave would be a contraction wave, which travels in the same direction as the robot's motion.

While $l(s, t)$ depends on the gait choice, the other backbone curve shape functions can be extracted from (23) by using the kinematic relationships

$$
T(s, t)=\sin ^{-1}\left(P_{3}^{\prime}(s, t) / l(s, t)\right)
$$

$$
K(s, t)=\operatorname{Atan} 2\left(\frac{P_{1}^{\prime}(s, t)}{l(s, t) \cos T(s, t)}, \frac{P_{2}^{\prime}(s, t)}{l(s, t) \cos T(s, t)}\right)
$$

where a " " denotes differentiation with respect to $s$.

Remark: Like the inextensible stationary wave gaits of Section V-B, these extension gait models also imply that the moving portion of the mechanism has less resistance to motion in the forward direction than in the reverse direction.

The rubbing and special friction assumption associated with the traveling wave extension gaits can be avoided by combining synchronous traveling waves of elongation and bending. In this approach, the shape functions that control bending assume the form of (7), while $l(s, t)$ assumes the form of (27), with $L_{w}$ the same in both cases. In this way the expanding segment is also bent to prevent it from sliding along the ground. For example, in the straight line locomotion case, shape functions of the form

$$
\begin{gathered}
l(s, t)=1+E_{l} W\left(s-\alpha_{t}(t), 0, L_{w}\right) \phi_{l}\left(s-\alpha_{t}(t)\right) \\
\theta(s, t)=E_{b} W\left(s-\alpha_{t}(t), 0, L_{w}\right) \phi_{t}\left(s-\alpha_{t}(t)\right)
\end{gathered}
$$

will implement this combined contraction and bending if $\phi_{t}(\cdot)$ is chosen so that $\theta(s, t)$ satisfies (13) and $\phi_{l}(\cdot)$ is chosen 

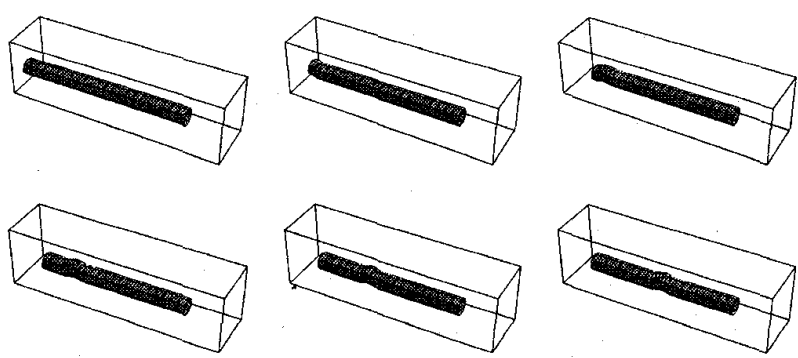

Fig. 11. Simulation of a gait using a combined bending and contraction wave.

so that $l(s, t)>0 \forall s$. An example of a locomotion gait employing (28) and (29) is shown in Fig. 11. For this case, $E_{l}=-0.5$ (i.e., contraction) and $E_{b}=0.2, \phi_{l}(\cdot)=1$, and $\phi_{t}\left(s-\alpha_{t}(t)\right)=\sin \left[\frac{2 \pi}{L_{w}}\left(s-\alpha_{t}(t)\right)\right]$. It can be shown that $L_{S L}=L_{w}\left[1-\left(1-E_{l}\right) J_{0}\left(E_{b}\right)\right]$.

\section{Choosing Among the Gaits}

The previous sections introduced a variety of gaits for implementing hyper-redundant locomotion. How does one select a gait for a given situation and a given robot mechanism? The answer to this question depends upon a large number of factors. These factors include the terrain geometry, the capabilities of the robot mechanism, the assumptions about friction between the robot and terrain, the necessary locomotion speed (defined as stride length per gait cycle), and the amount of power that can be supplied to the actuators. In this work we have focused on the macroscopic kinematics of a broad class of gaits, rather than on a particular actuation scheme required to implement locomotion. Thus, it is impossible to give strict gait choice guidelines for all possible hyperredundant mechanisms, as the guidelines are bound to be mechanism dependent. Here, we discuss some of the trade-offs which will naturally arise.

Our experience has shown that the stationary wave gaits (both extensible and inextensible) are most useful for paths which are nearly straight. The traveling wave gaits are to be preferred for paths with shaper bends.

Recall that the inextensible stationary wave gait and the extensible longitudinal traveling or stationary wave gaits all require special assumptions about the friction between the mechanism and the terrain. The surface of the mechanism in contact with the ground must have greater resistance to retrograde motion than forward motion. In Section IX we demonstrate one particular mechanism that implements this property. However, it may not be convenient or possible to outfit a robot with such devices. In such cases, the inextensible traveling wave gait, which does not require any special friction assumptions, can be used. While this gait is the most robust with respect to friction assumptions, it is one of the slowest gaits.

Many of the gaits could be implemented using a single traveling or stationary wave loop, or one could incorporate multiple waves. For a constant wave speed (in the case of traveling wave gaits) or constant oscillation period (in the case of stationary wave gaits), a multiple wave implementation of

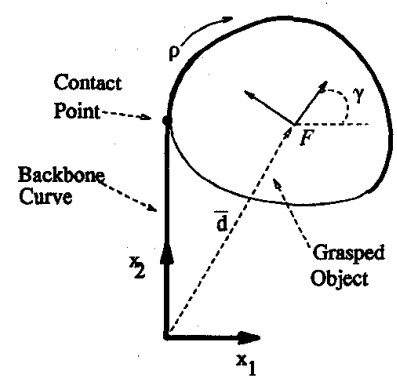

Fig. 12. Geometry of the tentacle-like grasp.

any of the gaits will typically result in faster forward robot speed. However, a multiple wave gait will obviously result in a greater actuator duty cycle and hence a greater power requirement. One would typically use smaller amplitude waves in a multiple wave implementation. This will lower the robot's center of mass, and thus lead to greater stability on highly uneven terrain.

\section{APPLICATIONS TO PLANNING THE MANIPULATION OF GRASPED OBJECTS}

These locomotion algorithms can also be used to plan the geometric aspects of a novel fine manipulation scheme for objects which are grasped in a tentacle-like fashion. A "massively redundant" tentacle-like grasp is considered in [20]. Here, we consider the novel combination of a hyperredundant grasp with a grasping wave scheme for reorienting the grasped object while maintaining a robust grasp. In effect, manipulation is accomplished by locomoting over the object boundary. This scheme is analogous to "finger gaiting" in multifingered robotic hand manipulation [15]. The fingers in this case are the contact points of the tentacle-like mechanism. This object manipulation scheme would be particularly useful for grabbing and manipulating a free flying satellite with a hyper-redundant robot arm. For simplicity, we only consider planar objects with convex boundary. Further, we only discuss the kinematic aspect of this object manipulation scheme, and not the important issues of force closure and internal mechanism force distribution in a tentacle-like grasp. That is, our goal is to illustrate that kinematic locomotion algorithms have other useful applications.

\section{A. Overview of Grasping Wave Method}

We assume that the robot mechanism's base is rigidly fixed. We further assume that the planar object to be grasped, $\mathcal{O}$, has a smooth convex boundary that can be described by an arc-length parametrized closed curve $\bar{o}(\rho)=\left[x_{1}(\rho), x_{2}(\rho)\right]^{T}$ in a coordinate frame, $\mathcal{F}$, attached to $\mathcal{O}$. The translation and rotation of $\mathcal{F}$ relative to the robot's base frame are respectively denoted by $\bar{d}(t) \in \mathbb{R}^{2}$ and $\gamma(t) \in S^{1}$ (Fig. 12). The object manipulation scheme consists of the following phases.

- Shape Initialization: The distal end of the mechanism wraps around $\mathcal{O}$ in a tentacle-like fashion. The section of the robot in contact with $\mathcal{O}$ is termed the grasp contact segment, $G_{c}$, and its associated backbone curve segment lies in the interval $s \in\left[s_{c}, 1\right]$. We assume that 
the length and geometry of $G_{c}$ is sufficient to enable force closure. The remainder of the backbone curve is termed the noncontact segment, $G_{n c}: s \in\left[0, s_{c}\right)$. $\bar{x}\left(s_{c}\right)$ is termed the contact point. The backbone curve is assumed to be tangent to $\mathcal{O}$ at $\bar{x}\left(s_{c}\right)$. For simplicity, we assume that at the end of shape initialization phase $\bar{x}\left(s_{c}\right)$ is located on the $x_{2}$-axis at a distance $h_{c}(t)$ from the robot base and that the tangent to $\mathcal{O}$ at $\bar{x}\left(s_{c}\right)$ points in the positive $x_{2}$ direction. We further assume that the boundary of $\mathcal{O}$ is parametrized such that increasing $s$ corresponds to increasing $\rho$. Let $\rho_{c}$ be the object boundary parameter at the grasp point. Thus, $\bar{x}\left(s_{c}\right)=$ $\bar{d}+\mathbf{R}(\gamma) \bar{o}\left(\rho_{c}\right)$, where $\mathbf{R}(\gamma)$ represents rotation by angle $\gamma$. The shape initialization phase is completed at time $t=0$.

- Phase 1: A section of the manipulator in $G_{n c}$ distorts to form a wave. This is analogous to stationary wave locomotion. This could be a bending, longitudinal, or combined wave. For inextensible mechanisms, $\mathcal{O}$ is necessarily displaced during this phase by the contraction of the backbone curve. This phase, whose duration is $t_{1}$, is shown in Fig. 13(a).

- Phase 2: The wave generated in Phase 1 travels along the mechanism toward the distal end (Fig. 13(b)). This phase is analogous to the traveling wave locomotion scheme of Section V-A. The "grasp terrain path," $\bar{P}_{G}$, consists of a fictitious path between the front of the grasp wave and $\bar{x}\left(s_{c}\right)$, as well as the boundary of $\mathcal{O}$ in $G_{c}$. If we assume that $G_{n c}$ is a straight line along the $x_{2}$-axis, then

$$
\begin{aligned}
\bar{P}_{G}(\mu, t) & =W\left(\mu, 0, h_{c}(t)\right)\left[\begin{array}{l}
0 \\
\mu
\end{array}\right]+W\left(\mu, h_{c}(t), 1\right)[\bar{d}(t) \\
& \left.+\mathbf{R}(\gamma(t)) \bar{o}\left(\mu-\rho_{c}(t)\right)\right] .
\end{aligned}
$$

Note that $\bar{P}_{G}$ is time varying, as several of its defining parameters will change from cycle to cycle. When the wave reaches the distal end of the robot, the arclength of $G_{c}$ will be longer by an amount $L_{S L}$. The period of this phase is $t_{2}-t_{1}$.

- Phase 3: By straightening the mechanism in the interval $s \in\left[s_{c}, s_{c}+s_{L}\right]$, the robot "unwraps" part of $G_{c}$ from $\mathcal{O}$ by an amount $L_{S L} . s_{L}$ is defined such that $L_{S L}=L\left(s_{c}+s_{L}, t\right)-L\left(s_{c}, t\right)$ (in the inextensible case $s_{L}=L_{S L}$ ). This results in a displacement of $\mathcal{O}$, and restores the length of $G_{c}$ to its original value. The duration of this phase is $t_{3}-t_{2}$.

When Phase 3 is complete, the cycle repeats, starting with Phase 1. This scheme results in repeated object rotations, whose magnitude depends on $L_{S L}$ and the shape of $\mathcal{O}$. One can compensate for the net translations of $\mathcal{O}$ which may occur from cycle to cycle by using the hyper-redundant robot endeffector placement methods in [8] and [9]. The cycle shown in Fig. 13 causes counter-clockwise rotations. The cycle can be reversed to yield clockwise rotations.

The grasping wave paradigm is easily illustrated for a disk. Following the assumptions above, the disc is initially grasped so that coordinates of its center are $\left(r_{c}, y_{c}\left(t_{0}\right)\right)$, where $r_{c}$ is the disc radius. Because of the disc's shape, $h_{c}(t)=y_{c}(t)$.
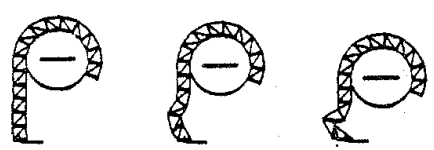

(a)
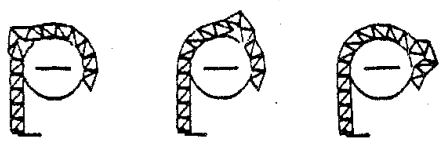

(b)
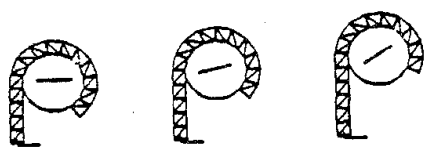

(c)

Fig. 13. The grasping wave object manipulation scheme.

$\bar{P}_{G}$ consists of a fictitious straight line segment between the robot's base and $\bar{x}\left(s_{c}\right)=\left(0, h_{c}(t)\right)$ and a circular arc along the object boundary

$$
\begin{aligned}
& \bar{P}_{G}(\mu, t)=W\left(\mu, 0, h_{c}(t)\right)\left[\begin{array}{l}
0 \\
\mu
\end{array}\right]+ \\
& W\left(\mu, h_{c}(t), 1\right)\left[\begin{array}{c}
r_{c}\left(1-\cos \left(\left(\mu-h_{c}(t)\right) / r_{c}\right)\right) \\
y_{c}(t)+r_{c} \sin \left(\left(\mu-h_{c}(t)\right) / r_{c}\right)
\end{array}\right] .
\end{aligned}
$$

For this example, the grasping wave is an inextensible traveling wave whose shape consists of three circular arc segments with respective arclengths $L_{w} / 4, L_{w} / 2$, and $L_{w} / 4$. The geometry of a planar inextensible backbone is strictly a function of $\theta(s, t)$, or equivalently, $\kappa(s, t)$. Here we choose to work with $\kappa(s, t)$ because of its simple expression for this object.

During the grasping wave cycle $\kappa(s, t)$ takes the form

$$
\begin{aligned}
\kappa(s, t)= & \kappa_{1}(s, t) W\left(t-I\left(t, t_{3}\right), 0, t_{1}\right) \\
& +\kappa_{2}(s, t) W\left(t-I\left(t, t_{3}\right), t_{1}, t_{2}\right) \\
& +\kappa_{3}(s, t) W\left(t-I\left(t, t_{3}\right), t_{2}, t_{3}\right) .
\end{aligned}
$$

That is, $\kappa_{1}, \kappa_{2}$, and $\kappa_{3}$ denote the backbone curve shape functions in the three different phases, and window functions are used to "section-off" these phases in time. During Phases 1 and 2 , the backbone curvature function takes the form

$$
\begin{aligned}
\kappa_{i}(s, t)= & W\left(s, h_{m}, 1\right)\left[1-W\left(s, s_{b}, s_{b}+L_{w}\right)\right] / r_{c} \\
& +\kappa_{\text {wave }} W\left(s, s_{b}, s_{b}+L_{w}\right)
\end{aligned}
$$

for $i=1,2$, where

$$
\begin{aligned}
\kappa_{\text {wave }}(s, t)= & -a_{1}(t) W\left(s, s_{b}(t), s_{b}(t)+\frac{L_{w}}{4}\right) \\
& +a_{2}(t) W\left(s, s_{b}(t)+\frac{L_{w}}{4}, s_{b}(t)+\frac{3 L_{w}}{4}\right) \\
& -a_{3}(t) W\left(s, s_{b}(t)+\frac{3 L_{w}}{4}, s_{b}(t)+L_{w}\right)
\end{aligned}
$$


is the grasp wave curvature, which consists of circular arcs. To form the grasping wave during Phase $1, a_{1}(t), a_{2}(t)$, and $a_{3}(t)$ take the monotonically increasing form

$$
a_{i}(t)=\frac{2 \pi}{L_{w} t_{1}}\left[t-I\left(t, t_{3}\right)\right] W\left(t-I\left(t, t_{3}\right), 0, t_{1}\right) \quad t \in\left[0, t_{1}\right]
$$

for $i=1,2,3$. This is analogous to part of a stationary wave gait cycle. Since the backbone is inextensible, the center of the disc is moved by a distance $L_{S L}=(1-2 / \pi) L_{w}$ in the negative $x_{2}$ direction during Phase 1 . In Phase $2, a_{1}(t), a_{2}(t)$, and $a_{3}(t)$ are computed using the numerical modal Jacobian approach of Section $\mathrm{V}$

$$
\begin{aligned}
& a_{i}(t)=W\left(t-I\left(t, t_{3}\right), t_{1}, t_{2}\right) \\
& \cdot\left(\frac{2 \pi}{L_{w}}+\int_{t_{1}+I\left(t, t_{3}\right)}^{t} \sum_{k=1}^{3} J_{i k}^{-1}\left(\Delta \dot{\bar{X}}_{k}^{\mathrm{des}}-\frac{\partial \Delta \bar{X}_{k}(\bar{a}, t)}{\partial t}\right) d t\right)
\end{aligned}
$$

where $\Delta \bar{X}^{\text {des }}(t)$ is computed from $\bar{P}_{G}$. In this way, the grasping wave tracks the grasp terrain, like a traveling wave locomotion gait.

In Phase 3 the curvature function assumes the form

$$
\kappa_{3}(s, t)=W\left(s, h_{m}(t), 1\right) / r_{c},
$$

to implement the "unwrapping" of the object. $h_{m}(t)$ is a function that monotonically increases from the value $h_{m}\left(t_{2}\right)=$ $y_{c}(0)-L_{w}(1-2 / \pi)$ to $h_{m}\left(t_{3}\right)=y_{c}(0)$.

Note that this example was used in the computer simulation which generated Fig. 13, with the addition of a fitting process to adapt the discretely segmented variable geometry truss structure to the continuous backbone curve solution. Additional examples using other wave shapes and wave types can be found in [4].

\section{EXPERIMENTAL DEMONSTRATION OF HYPER-REDUNDANT LOCOMOTION AND GRASPING}

To check our modeling assumptions, verify the correctness of these algorithms and validate their usefulness, several of the locomotion gaits and the object manipulation scheme have been implemented in an actual 30 degree-of-freedom hyperredundant robot system. A more detailed description of this system can be found in [6]. Briefly, this system (Fig. 14) is a planar VGT structure consisting of 10 identical modules, where each module is a planar 3 DOF parallel manipulator. The prismatic actuators are implemented with dc servo motors and lead screw drives. The actuators can vary in length from a minimum of 12 in to a maximum of $18 \mathrm{in}$. The robot system is controlled by a multiprocessing computer, which consists of Heurikon 68030 processing boards in a VME bus, which is in turn connected to a Sun Microsystems 4/260 computer.

For grasping and object manipulation experiments, the robot operates in the plane of the laboratory floor (where casters allow low friction rolling over the floor). For locomotion experiments, the manipulator is detached from its base, and turned on its side so that it can locomote in a vertical plane. Each module is equipped with rubber faced ratchet wheels

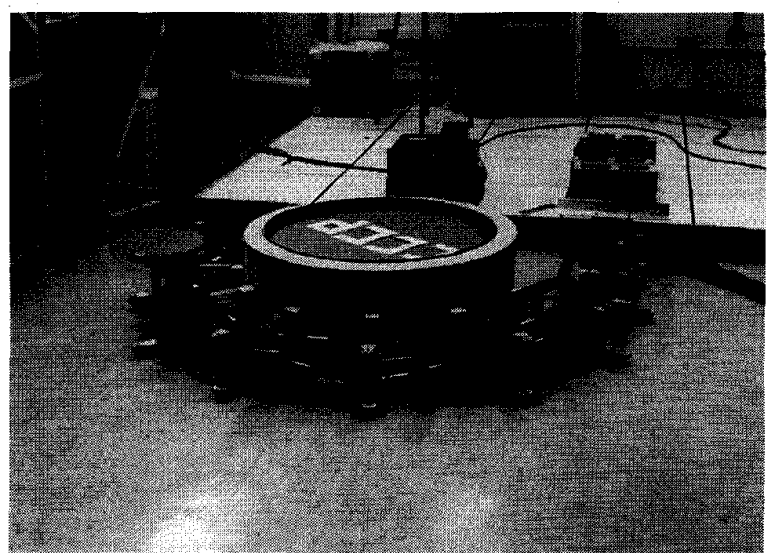

Fig. 14. Picture of VGT hyper-redundant system grasping a disc (mock "satellite").

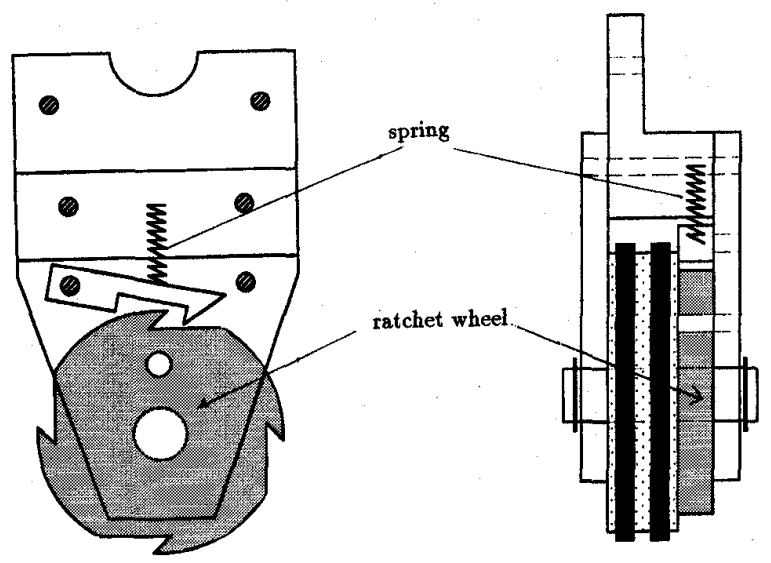

Fig. 15. Schematic of ratchet wheel that implements special friction properties. Left view is schematic of ratchet mechanism, which has 6 teeth in the real device, not 4 as shown. Right View is cross section of wheel and ratchet.

(Fig. 15) which permit rolling in one direction only. These wheels perform the same function as scales on a snake-they prevent retrograde motion while easily permitting forward motion. As discussed in Sections V-B and VI, these devices are required for some gaits. For traveling wave locomotion, the wheels are locked in place, and the wheels' rubber surfaces are used as passive feet. While the devices are currently constructed to have a fixed preferred direction of motion, an electronic brake could easily be added so that the preferred direction of motion can be changed under computer control. Because of its modularity, the robot can be separated into two 15 DOF robots for implementing locomotion with a smaller (e.g., fewer degrees of freedom) robot.

Most of the locomotion gaits presented in this paper have been implemented and verified with this device. For example, Fig. 16 shows a sequence of snapshots during an experiment in which the robot uses a stationary bending wave locomotion gait to navigate a straight line on flat ground. While this robot is extensible, in this experiment we arbitrarily restrict it to use an inextensible gait. This experiment exactly implements the shape function of (20), for the case $m=2$, with the fitting algorithms in [8]. Recall from Section V-B. that this gait requires the unique properties of the ratchet wheels. 


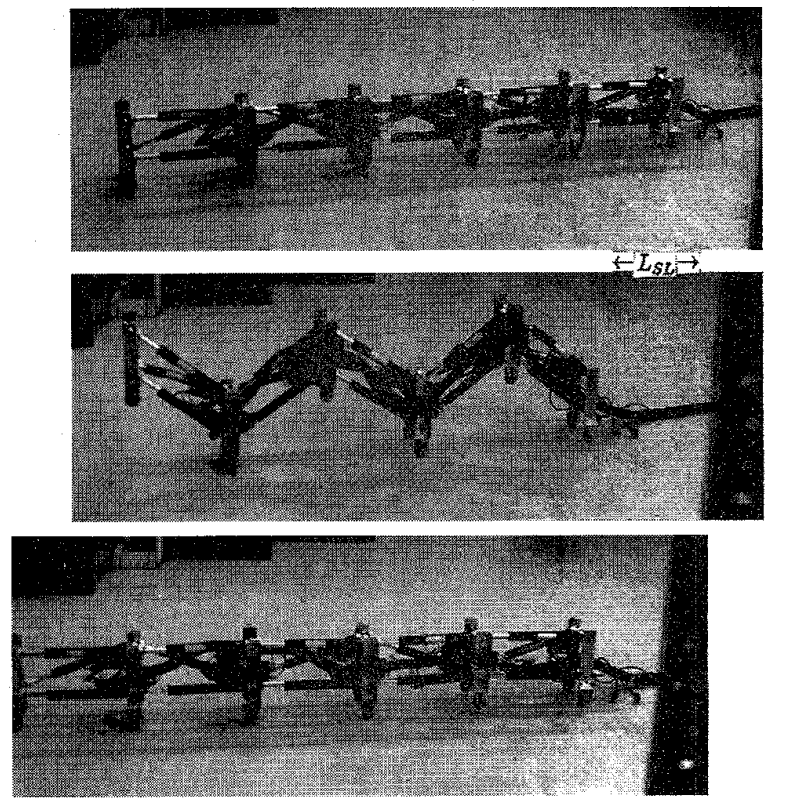

Fig. 16. Inextensible stationary wave locomotion experiment. Top: beginning of cycle. Middle: midpoint of gait cycle. Bottom: end of cycle. The robot moves to the left.
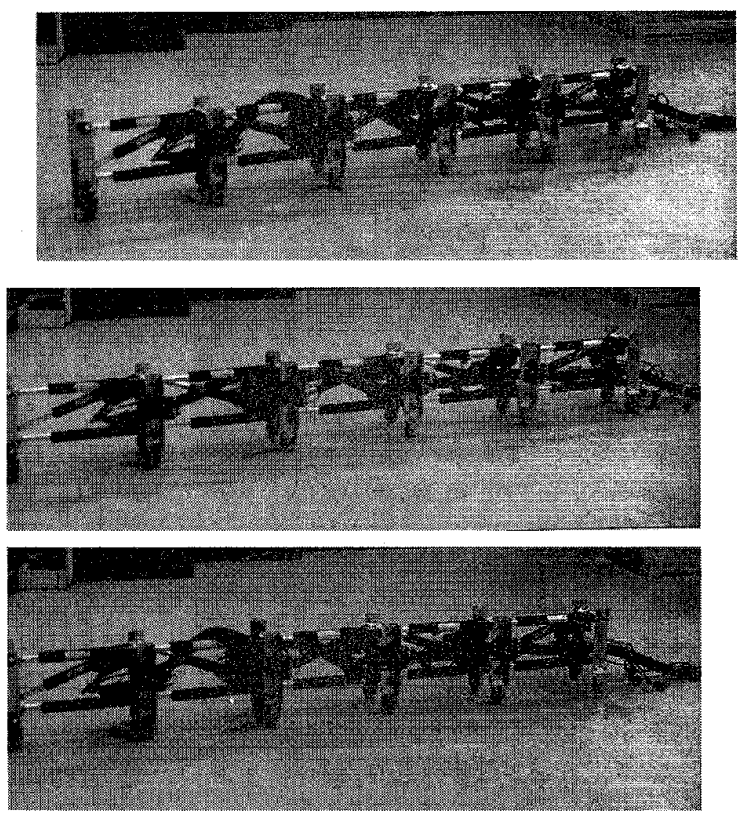

Fig. 17. Longitudinal stationary wave locomotion. Top: beginning of cycle with robot in contracted state. Middle: approximate midpoint of cycle, with robot extended. Bottom: end of cycle. The robot moves to the left.

Similarly, Fig. 17 shows snapshots of a longitudinal stationary wave locomotion experiment which employs the methods of Section VI. In this case, the backbone curve shape functions assume the form of (24) with $\phi_{s}=1$ and $a_{s}(t)$ having the form of (20). Fig. 18 depicts a longitudinal traveling wave locomotion experiment that uses a contraction wave, as opposed to expansion wave of the simulation in Fig. 10. Here again, these gaits also require the special properties of the ratchet wheels.
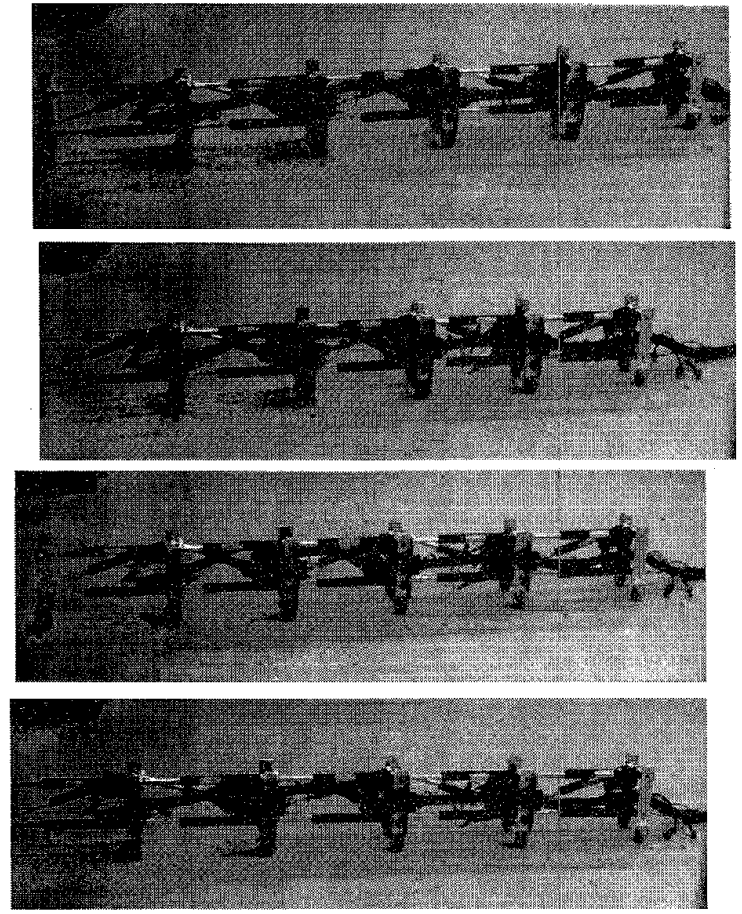

Fig. 18. Traveling contraction wave locomotion. The robot travels to the left using a contraction wave which also travels to the left. Top: beginning of cycle-note reference markers on the ground next to the wheels. Bottom: end of cycle.

Fig. 14 shows how a hyper-redundant manipulator arm can be used to envelop an object, which in this instance is a mock satellite. The backbone curve shape equations developed in the example of Section VIII were used in this experiment to manipulate the "satellite." It is difficult and lengthy to present the grasping wave object manipulation experiment in this paper. Interested readers can find a record of this sequence of manipulations in a portion of the video of [7].

It should be noted that because of the computational efficiency of the algorithms presented in this paper, only a single Motorola 68030 processor was required to compute the locomotion and motor servo algorithms for all 30 actuators. Thus, our experimental work verifies the fact that hyperredundant locomotion can be implemented with relatively little computational power when using the formulation presented in this paper. Further, the schemes are quite robust, as the mechanical ratchet wheels enforce the desired friction constraints between the robot and the ground over a broad range of the coefficient of friction between the robot and the ground. That is, no special properties of the laboratory floor were required to effect locomotion, and locomotion was successful over the entire range of speeds which are achievable by the mechanism.

\section{CONCLUSTON}

This paper has shown that a large number and variety of hyper-redundant robot locomotion gaits can be understood and implemented using simple stationary and/or traveling wave patterns of mechanism bending and extension. Further, we showed how these locomotion gaits can be used to implement 
a novel scheme for manipulating objects which are grasped in a tentacle-like fashion. We modeled the kinematics of hyperredundant locomotion via a continuous backbone curve model. This abstraction allowed us to clearly focus on the important macroscopic geometric patterns of mechanism displacement which are the key to hyper-redundant robot locomotion, without having to focus on a particular hyper-redundant robot mechanical implementation. The implementation details can often be relegated to a "fitting" procedure. The analysis of dynamics and friction effects on locomotion behavior require more explicit and complicated models than the ones presented here. However, these models are bound to depend upon the characteristics of a particular mechanism and gait. In this paper, we chose instead to focus on the macroscopic kinematic behavior of a large class of gaits, rather than a detailed analysis of one particular gait or mechanism.

Our experimental implementation of these algorithms indicates that they are practical and implementable with a very reasonable amount of computing power. Our experiments also verified that our assumptions on friction between the robot and the terrain could easily be achieved using simple mechanical devices and that these assumptions were realistic in practice. We thus conclude that the algorithms and experiments described in this paper are a step toward making hyperredundant robot systems more widely used.

\section{REFERENCES}

[1] J. C. Alexander and J. H. Maddocks, "On the kinematics of wheeled mobile robots," Int. J. Robot. Res., vol. 8, no. 5, pp. 15-27, 1989.

[2] V. V. Anderson and R. C. Horn, "Tensor-arm manipulator design," ASME Trans., vol. 67-DE-57, pp. 1-12, 1967.

[3] S. Bennet, T. McConnel, and S. L. Trubatch, "Quantitative analysis of the speed of snakes as a function of peg spacing," J. Exp. Biology, vol. 60 , pp. 161-165, 1974.

[4] G. S. Chirikjian, "Theory and applications of hyper-redundant robotic manipulators," Ph.D. dissertation, Dep. Appl. Mechanics, California Inst. Technol., Pasadena, June, 1992.

[5] G. S. Chirikjian and J. W. Burdick, "An obstacle avoidance algorithm for hyper-redundant manipulators," in Proc. IEEE Int. Conf. Robotics and Automation, Cincinnati, OH, May 14-17, 1990, pp. 625-631.

[6] ____, "Design and experiments with a 30 degree-of-freedom robot," in Proc. IEEE Int. Conf Robotics and Automation, Atlanta, GA, May 2-5, 1993, vol. 3, pp. 113-119.

[7] _ "Experiments in hyper-redundant manipulation," in Video Proc. IEEE Int. Conf. Robotics and Automation, Atlanta, GA, May 2-5, 1993.

[8] - "A modal approach to the kinematics of hyper-redundant manipulators," IEEE Trans. Robot. Automat., vol. 10, no. 3, pp. 343-354, June 1994

[9] , "Kinematically optimal hyper-redundant manipulator configurations," IEEE Trans. Robot. Automat, vol. 11, no. 6, pp. 794-806, Dec. 1995.

[10] M. P. DoCarmo, Differential Geometry of Curves and Surfaces. Englewood Cliffs, NJ: Prentice-Hall, 1976.

[11] R. T. Farouki and C. A. Neff, "Analytical properties of plane offset curves," Comput. Aided Geometric Design, vol. 7, pp. 83-99, 1990.

[12] T. Fukuda, H. Hosokai, M. Uemura, "Rubber gas actuator driven by hydrogen storage alloy for in-pipe inspection mobile robot with flexible structure," in Proc. IEEE Int. Conf. Robotics and Automation, Scottsdale, AZ, May 1989, pp. 1847-1852.
[13] S. Hirose and A. Morishima, "Design and control of a mobile robot with an articulated body," Int. J. Robot. Res., vol. 9, no. 2, pp. 99-114, 1990.

[14] S. Hirose and Y. Umetani, "Kinematic control of active cord mechanism with tactile sensors," in Proc. 2nd Int. CISM-IFT Symp. Theory and Practice of Robots and Manipulators, 1976, pp. 241-252.

[15] J. Hong et al., "Fine manipulation with multifinger hands," in Proc. IEEE Int. Conf. Robotics and Automation, Cincinatti, OH, 1990, pp. 1568-1573.

[16] B. C. Jayne, "Kinematics of terrestrial snake locomotion," Copeia, no. 4, pp. $915-927,1986$.

[17] H. D. Jones, "The mechanism of locomotion of Agriolimax reticulatus (Mollusca: Gastropoda)," J. Zoology London, vol. 171, pp. 489-498, 1973.

[18] J. B. Keller and M. S. Falkovitz, "Crawling of worms," J. Theor. Biol., vol. 104 , no. 3, pp. $417-442,1983$.

[19] H. W. Lissmann, "Rectilinear locomotion in a snake (Boa occidentalis)," J. Exp. Biology, vol. 26, pp. 368-379, 1950.

[20] J. S. Pettinato and H. E. Stephanou, " Manipulability and stability of a tentacle based robot manipulator," in Proc. IEEE Int. Conf. Robotics and Automation, Scottsdale, AZ, May 15-19, 1989, pp. 458-463.

[21] M. H. Raibert, Legged Robots That Balance. Cambridge, MA: MIT Press, 1986.

[22] M. K. Seymour, "Locomotion and coelomic pressure in Lumbricus Terrestris L.," J. Exp. Biology, vol. 51, pp. 47-58, 1969.

[23] S. M. Song and K. J. Waldron, Machines That Walk. Cambridge, MA: MIT Press, 1989.

[24] J. R. Stulce, W. E., Burgos, S. G. Dhande, and C. F. Reinholtz "Conceptual design of a multibody passive-legged crawling vehicle," in Proc. ASME Mechanisms Conf., Chicago, IL, Sept. 16-19, 1990, DE-vol. 26, pp. 199-205.

[25] W. B. Yapp, "Locomotion of worms," Nature, London, vol. 177, pp. 614-615, 1956

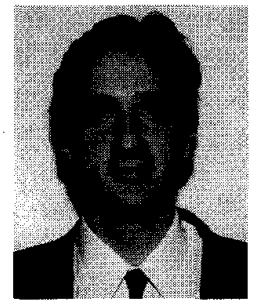

Gregory S. Chirikjian (M'93) received the B.S.E. degree in engineering mechanics and the M.S.E. degree in mechanical engineering in 1988 while also fulfilling requirements for the B.A. degree in mathematics, all from Johns Hopkins University, Baltimore, MD. Between 1988 and 1992 he was a graduate student at the California Institute of Technology, Pasadena, where he received the Ph.D. degree in applied mechanics.

Since the summer of 1992 , he has been with the Department of Mechanical Engineering at Johns Hopkins as an Assistant Professor, where he started the robotics program. His general interests are in the kinematic analysis, design, and implementation of "hyper-redundant," "metamorphic," and "binary" manipulators.

Dr. Chirikjian received an NSF National Young Investigator Award through the Robotics and Machine Intelligence Program in 1993 and a Presidential Faculty Fellows Award in 1994 to continue work in these areas.

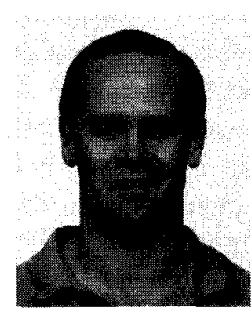

Joel W. Burdick received the B.S.M.E. degree from Duke University, Durham, NC, in 1981, and the Ph.D. degree in mechanical engineering from Stanford University, Stanford, CA, in 1988

In May 1988, he was with the Department of Mechanical Engineering, California Institute of Technology, Pasadena, as an Assistant Professor, where he is currently an Associate Professor. His general research interests are in hyper-redundant robots, medical robots, dynamically stable legged locomotion, and kinematic mobility theory.

Dr. Burdick is a National Science Foundation Presidential Young Investigator, an Office of Naval Research Young Investigator, and the Hughes Feynman Fellow. 